https://helda.helsinki.fi

\title{
The cognitive neuroscience of auditory distraction
}

\section{Campbell, Thomas}

2005

Campbell , T 2005 , ' The cognitive neuroscience of auditory distraction ' , Trends in

Cognitive Sciences , vol. 9 , no. 1 , pp. 3-5 . https://doi.org/10.1016/j.tics.2004.11.002

http://hdl.handle.net/10138/161136

https://doi.org/10.1016/j.tics.2004.11.002

publishedVersion

Downloaded from Helda, University of Helsinki institutional repository.

This is an electronic reprint of the original article.

This reprint may differ from the original in pagination and typographic detail.

Please cite the original version. 
5 Kšhler, W. and Wallach, H. (1944) Figural aftereffects: an investigation of visual processes. Proc. Am. Philos. Soc. 88, 269-357

6 Gepshtein, S. and Kubovy, M. Stability and change in perception: spatial organization in temporal context. Exp. Brain Res. (published online ahead of print)

7 Kubovy, M. (1994) The perceptual organization of dot lattices. Psychon. Bull. Rev. 1, 182-190

8 Kubovy, M. and Wagemans, J. (1995) Grouping by proximity and multistability in dot lattices. Psychol. Sci. 6, 225-233

9 Kubovy, M. et al. (1998) On the lawfulness of grouping by proximity. Cogn. Psychol. 35, 71-98

10 Hock, H.S. et al. (1996) Perceptual stability and the selective adaptation of perceived and unperceived motion directions. Vision Res. 36, 3311-3323

11 Hock, H.S. et al. (1993) Bistability and hysteresis in the organization of apparent motion patterns. J. Exp. Psychol. Hum. Percept. Perform. $19,63-80$

12 Kruse, P. et al. (1996) Continuous phase transitions in the perception of multistable visual patterns. Biol. Cybern. 75, 321-330
13 Borsellino, A. et al. (1972) Reversal time distribution in the perception of ambiguous stimuli. Kybernetik 10, 139-144

14 Zhou, Y.H. et al. (2004) Perceptua l dominance time distributions in multistable visual perception. Biol. Cybern. 90, 256-263

15 Blake, R. et al. (2003) Visual motion retards alternations between conflicting perceptual interpretations. Neuron 39, 1-20

16 Riani, M. and Simionotto, E. (1994) Stochastic resonance in the perceptual intepretation of ambiguous figures. Phys. Rev. Lett. 72, 3120-3123

17 Merk, I. and Schnakenberg, J. (2002) A stochastic model of multistable visual perception. Biol. Cybern. 86, 111-116

18 Kanizsa, G. and Gerbino, W. (1976) Convexity and symmetry in figure-ground organization. In Vision and Artifact (Henle, M. ed.), pp. 25-32, Springer

19 Leopold, D.A. et al. (2002) Stable perception of visually ambiguous patterns. Nat. Neurosci. 5, 605-609

1364-6613/\$ - see front matter (c) 2004 Elsevier Ltd. All rights reserved. doi:10.1016/j.tics.2004.11.001

\title{
The cognitive neuroscience of auditory distraction
}

\section{Tom Campbell $1,2,3$}

\author{
${ }^{1}$ Helsinki Collegium for Advanced Studies, PO Box 4, FIN-00014 University of Helsinki, Finland \\ ${ }^{2}$ Biomag Laboratory, Engineering Centre, Helsinki University Central Hospital, Finland \\ ${ }^{3}$ Helsinki Brain Research Centre, University of Helsinki, Finland
}

\begin{abstract}
We are often aware of the content of distracting sound, although typically remain unaware of the processes by which that sound is disruptive. Disruption can occur even when the sound is ignored and unrelated to the task being performed. In a recent major development, Gisselgård et al. have used positron emission tomography to reveal how distracting sounds recruit the involvement of dorsolateral prefrontal cortex.
\end{abstract}

When our mental activities are the most demanding is often when we become most aware of the distracting influences of background sound. Working memory is a function of the brain that permits the short-term maintenance of information that needs to be remembered. The manipulation of that maintained information within working memory is often used in the service of a particular task or goal. It is the mental activities that place heavy demands upon working memory that seem to be most susceptible to the disruptive effects of auditory distraction. Instances of such mental activities include reading, arithmetic or (in laboratory experiments) silently reading a list of numbers and reporting back that series after a brief delay [1,2]. Recently, Gisselgård, Petterson and Ingvar $[3,4]$ have revealed that for auditory distraction to disrupt working-memory performance requires the activation of the dorsolateral prefrontal cortex of the brain. Indeed, this crucial activation was only seen to occur on a difficult working-memory task [4].

Corresponding author: Campbell, T. (tomcampbell@mariecurie.org).
When we succeed in ignoring - suppression of a largescale network of brain areas

Change within the ignored distracting sound has been pinpointed as a key determinant of disruption of working memory by auditory distraction ([5,6], see also [7]). That is, a changing-state sequence of sounds (e.g. ABAB...) typically proves more disruptive than a steady-state sequence of ignored speech sounds (e.g. AAA...). To explain this changing-state effect, cognitive theory has invoked the concept of an involuntary processing of ignored changingstate material, which disrupts the processing of the to-beremembered material [5]. This changing-state auditory distraction might be related to particular brain processes [8-11] although the functional anatomy of these processes have remained yet to be fully understood.

Two PET experiments conducted by Gisselgård et al. [3,4] shed considerable light on the functional anatomy of the crucial brain processes by contrasting the action of ignored steady-state and changing-state speech sound during a working-memory task. This series of experiments not only investigated the effects of different types of speech sound on the accuracy of performance on a task, the working-memory performance, but also used PET to measure regional cerebral blood flow under conditions of steady-state and changing-state auditory distraction. Increases in blood flow in a region were interpreted to reflect metabolism within that brain region (activation), whereas decreases reflected the suppression of that metabolism (deactivation).

The working-memory task - immediate verbal serial recall - entailed the visual presentation of a list of 6 digits 
in random order (e.g. 2-7-4-6-3-1) one at a time, after which volunteers were required to report back the digit items in the correct order [3]. The approach adopted was to compare the state of the brain on this easy workingmemory task with that on a control task with minimal working-memory requirements. This control task entailed presentation of a list of the digits 1-6 in numerical order (1-2-3-4-5-6) one at a time, after which volunteers were required to report back the digit items in the correct order. This comparison revealed a working-memory effect characterised by an activation of a large-scale network of brain regions.

This network consisted of an activation of a large number of cortical regions (left inferior frontal cortex, left anterior cingulate cortex, left inferior parietal cortex, right precuneus, bilateral anterior insular cortex), as well as subcortical regions (left lentiform nucleus, left thalamus and bilateral cerebellum). There were also deactivations of several cortical regions (bilateral inferior prefrontal cortex, bilateral medial prefrontal cortex, right posterior cingulate cortex, right superior temporal cortex and right middle/superior temporal cortex).

The lateral prefrontal cortex was a component of the activated network, in corroboration of the involvement of this region in working-memory function [12]. The upper portion of the lateral prefrontal cortex, is termed dorsolateral (BA 9 and 46) and the lower portion is termed ventrolateral (BA 44, 45 and 47) (see Figure 1). Indeed, this anatomical distinction also loosely determines the working-memory functions of this cortex such that: (i) ventrolateral prefrontal cortices are involved in the maintenance of to-be-remembered information (e.g. retaining the order of a short list of letters); (ii) dorsolateral prefrontal cortices are involved in the manipulation of that information (e.g. alphabetical re-ordering of a short list of letters) [13,14]. Whereas Gisselgård et al.'s [3] working-memory effect activated the left inferior frontal cortex, (BA 6/44), which constitutes part of the ventrolateral prefrontal 'maintenance' cortex, the dorsolateral prefrontal 'manipulation' cortex was not activated.

This working-memory effect on the brain varied such that, relative to steady-state material, change-of-state caused a deactivation of several of the regions involved in working memory. However, these effects of changing-state sound on the brain were not shown to adversely influence working-memory performance on this task. Changingstate material resulted in a suppression of several brain areas, crucially involving left ventrolateral prefrontal cortex and brain regions implicated in the temporary storage of speech-based material: bilateral secondary auditory and left inferior parietal cortex.

These regions, which are involved in working memory, processed the changing-state sound, suppressing the effects of that sound, and so successfully ignored that sound such that working-memory performance was unaffected. When auditory distraction disrupts working peformance on tasks with similar low memory demands (e.g. [15]), it remains an open question whether this disruption is related to the absence of suppression or the activation of some additional brain mechanism. Arguably,

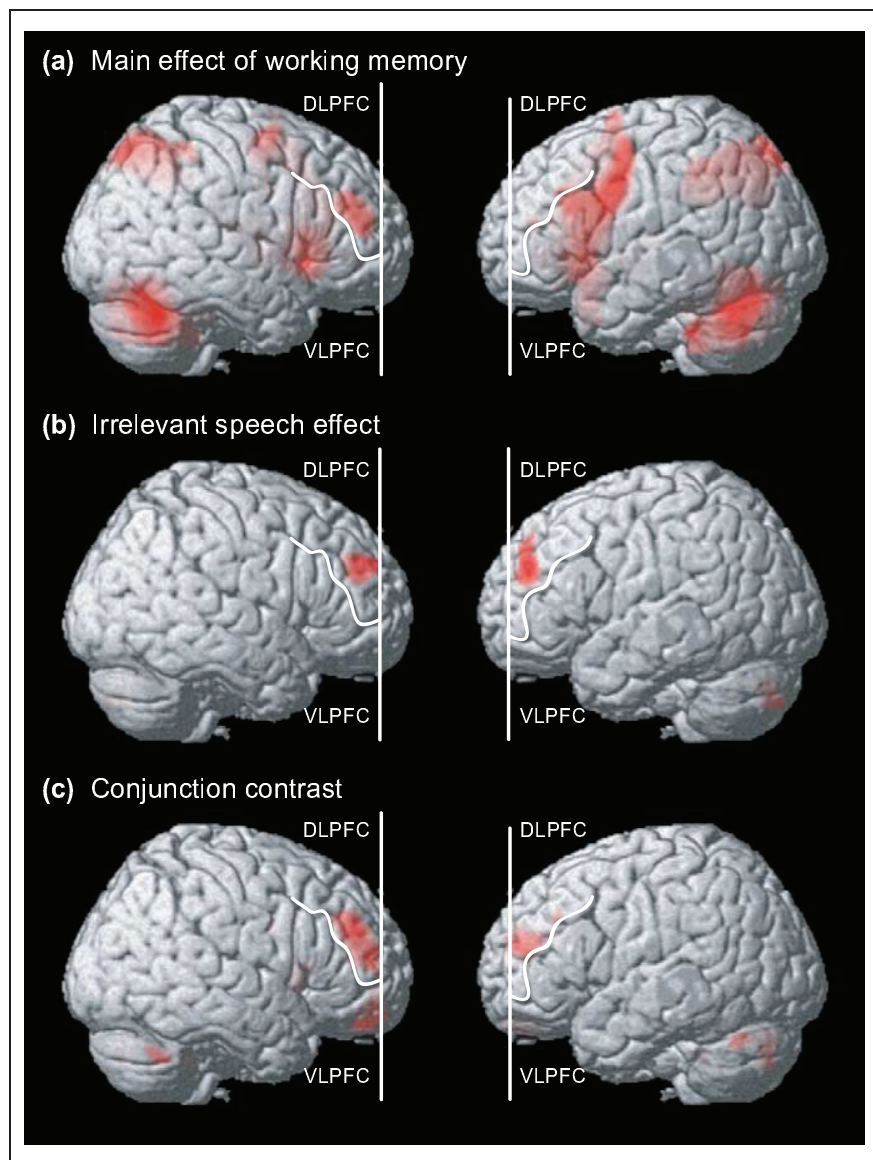

Figure 1. (a) Increases of blood flow associated with the main effect of working memory. (b) Related increases of blood flow associated with the influence of changing versus steady-state sounds are confined to the dorsolateral prefrontal cortices. (c) Commonalities of the above. The division between dorsolateral prefrontal cortex (DLFPC) and ventrolateral prefrontal cortex (VLPFC) is indicated by the white curved line. The vertical white line indicates the anterior boundary of lateral prefrontal cortex. Reproduced with permission from [4].

in Gisselgård et al.'s study [3] the presence of this suppression precluded the activation of brain mechanisms that, otherwise, would produce a disruption of workingmemory performance.

In this regard, it is worth considering that, with this task, the dorsolateral prefrontal 'manipulation' cortex was neither involved in the effects of working memory nor that of changing-state.

\section{When we cannot ignore - activation of dorsolateral prefrontal cortex}

Now, with a near-identical working-memory procedure, ignored speech sounds disrupted working-memory performance [4]. In this condition, the number of to-beremembered items was increased to 8 rather than 6 digits per list. The $10 \%$ decrease in baseline performance (6 digits: $93 \%$ correct, compared with 8 digits: $83 \%$ correct) on this more difficult task might be one of the reasons why this task was more susceptible to disruption. What was it about the activation of the brain during this more difficult task that permitted a disruption of working-memory performance by auditory distraction?

The additional demands of the task recruited the involvement of the dorsolateral prefrontal 'manipulation' cortex on this difficult working-memory task [4]. As shown 
in Figure 1a, the working-memory effect replicated the same pattern of activations as was seen in the previous PET procedure, including that of the ventrolateral prefrontal 'maintenance' cortex. Additional activations, not seen with the easier task [3] were also shown in anterior and medial prefrontal cortex, but of most theoretical interest was the additional involvement of the right dorsolateral prefrontal 'manipulation' cortex.

Comparing working-memory effects under circumstances of changing-state and steady-state sound (see Figure 1b) revealed that this changing-state effect also activated the dorsolateral prefrontal cortices. As shown in Figure 1c, similarities between the brain regions activated by working-memory and changing-state effects were confined to the dorsolateral prefrontal 'manipulation' cortex. The dorsolateral prefrontal cortices are thus activated by the higher memory demands of this difficult task and are also additionally activated by changing-state sound. Arguably, this distracting sound thus crucially influences the processes of executive functioning within the brain $[12,16]$ that support the active selection, monitoring and manipulation of information in working memory.

\section{Implications for future research}

With an easy task, auditory distraction is prevented from disrupting working memory by a physiological suppression of the effects of the changing-state sound. This physiological suppression might reflect a suppression of the content of the irrelevant material, preventing that material from adversely affecting working-memory performance on easy tasks. Indeed, on the difficult task, when ignored speech disrupted working-memory performance [4], this physiological suppression was not apparent.

Arguably, we are often aware of the content of distracting sound when that sound disrupts our performance. However, we are typically unaware of the processing that produces this distraction. With a difficult task, a disruption of working-memory performance is seen that might be related to the brain mechanisms involved in the conscious processing of material held in working memory. This processing crucially involves the dorsolateral prefrontal cortex [4].

Changing-state disruption of working-memory peformance occurs not only with to-be-remembered material of visual-verbal content, such as digits, but also with material of visual-spatial content, such as the positions of dots [17]. Seemingly then, auditory distraction is one of process rather than content. An interesting question thus remains to be answered: when the content of the to-be- remembered material is visual-spatial, is the involvement of the dorsolateral prefrontal cortex crucial to the disruption of working-memory peformance?

\section{Acknowledgements}

Tom Campbell is supported by the University of Helsinki. Thanks are due to Jens Gisselgård, MR Centre, Karolinska Institute, Stockholm, for communication concerning this work and for Figure 1.

\section{References}

1 Banbury, S. and Berry, D.C. (1997) Habituation and dishabituation to speech and office noise. J. Exp. Psychol. Appl. 3, 1-16

2 Banbury, S. and Berry, D.C. (1998) The disruption of office-related tasks by speech and office noise. Br. J. Psychol. 89, 285-295

3 Gisselgård, J. et al. (2003) The irrelevant speech effect: a PET study. Neuropsychologia 41, 1899-1911

4 Gisselgård, J. et al. (2004) The irrelevant speech effect and working memory load. Neuroimage 22, 1107-1116

5 Jones, D.M. et al. (1996) The object-oriented episodic record model. In Models of Short-Term Memory (Gathercole, S.E., ed.), pp. 209-238, Psychology Press

6 Jones, D.M. et al. (2004) The phonological store of working memory: Is it phonological and is it a store? J. Exp. Psychol. Learn Mem. Cogn. 30, 656-674

7 Campbell, T.A. et al. (2002) Auditory memory and the irrelevant sound effect: Further evidence for changing-state disruption. Memory 10, 199-214

8 Campbell, T.A. et al. (2003) The N1 hypothesis and irrelevant sound: Evidence from token set effects. Brain Res. Cogn. Brain Res. 18, 39-47

9 Valtonen, J. et al. (2003) Visual short-term memory load affects sensory processing of irrelevant sounds in human auditory cortex. Brain Res. Cogn. Brain Res. 17, 358-367

10 Kopp, F. et al. (2004) Neural networks engaged in short-term memory rehearsal are disrupted by irrelevant speech in human subjects. Neurosci. Lett. 354, 42-45

11 Hadlington, L. et al. (2004) Auditory location in the irrelevant sound effect: The effects of presenting auditory stimuli to either the left ear, right ear or both ears. Brain Cogn. 55, 545-557

12 Fuster, J.M. (1997) The Prefrontal Cortex: Anatomy, Physiology and Neuropsychology of the Frontal Lobe, Lippincott-Raven

13 Fletcher, P.C. and Henson, R.N.A. (2001) Frontal lobes and human memory - insights from functional imaging. Brain 124, 849-881

14 Postle, B.R. et al. (1999) Functional neuroanatomical double dissociation of mnemonic and executive control processes contributing to working memory performance. Proc. Natl. Acad. Sci. U. S. A. 96, 12959-12964

15 Salame, P. and Baddeley, A.D. (1986) Phonological factors in STM: similarity and the unattended speech effect. Br. Psychonomic Soc. 24, 263-265

16 Baddeley, A.D. (1986) Working Memory, Clarendon Press

17 Jones, D.M. et al. (1995) Functional equivalence of verbal and spatial information in serial short-term-memory. J. Exp. Psych. Learn Mem. Cogn. 21, 1008-1018

1364-6613/\$ - see front matter (c) 2004 Elsevier Ltd. All rights reserved. doi:10.1016/j.tics.2004.11.002 